\title{
Simulations of Lévy Walk
}

\author{
Venkat Abhignan $^{1}$ (1) $\cdot$ Sinduja Rajadurai ${ }^{2}$
}

Received: 20 July 2020/Accepted: 3 February 2021 / Published online: 30 March 2021

(C) The Institution of Engineers (India) 2021

\begin{abstract}
The simulation of stable distributions was performed to study an ideal movement pattern for the spread of a virus using an autonomous carrier. It has been observed that Lévy walks are the most ideal way to spread and further study was done on how the parameters in Lévy distribution affect the spread.
\end{abstract}

Keywords Stable distributions - Lévy walk - Simulations

\section{Introduction}

Biological systems cannot be described only in the aspects of probabilistic distributions. However, in the interest of studying interactions and common behaviours we consider systems to imitate similar laws at different scales [1]. Stable distributions have scale invariant behaviour, where linear combination of independent and identical distributions (IIDs) with finite mean and variance leads to a normal distribution (ND) [2]. Similarly, when rescaled and reordered sum of IIDs were considered with non-finite variance, it may converge to a Lévy distribution (LD). Though NDs are pervasive in most systems, LDs are found in biological system mostly associated with optimal foraging behaviour [1,3] and human mobility. Most literature supports the statistical similarity between human mobility through different modes of transportation and Lévy distribution [4-6], but in some instances a log-normal

Venkat Abhignan

yvabhignan@gmail.com

1 National Institute of Technology Tiruchirappalli, Tiruchirappalli, India

2 Tiruchirappalli, India distribution was also observed [7]. LD comprises Lévy walk (LW) where multiple short steps are taken with long steps in-between, whereas the ND comprises Brownian walk (BW) where multiple similar steps are taken. Observing the spread of present virus [8], studying simulations have been considered of a simple model using stable distributions. It can be observed that spread takes a Lévy like walk which seems to happen in different distance scales. Initially, spreading across different continents taking long steps followed by multiple short steps within the continent, then again long steps across different countries within the continent and followed by multiple short steps within the country, then different states within the country and so on, the distance scales keep changing but the behaviour is nearly the same. This similar behaviour can perhaps be observed in the recently available data of COVID-19 taken up to 10th November 2020 [8].

Fig 1(a) shows the data of daily new cases in different countries, and Fig 2(a) shows the data of daily new cases per 100,000 people in different states within the USA. It has been observed that the different distributions of these countries and states are characterized by an initial peak followed by a decline or flattening of the curve and then followed by a higher peak than the initial. It was assumed that the macroscopic behaviour of such a system is nearly the same which can be a continent, or a country within the continent, or a state within the country. Independent of the microscopic interactions which influence the local behaviour such as environmental factors, demographics, government policies implemented to control the spread and various other factors that are different in each scale and each system. This study qualitatively concerns only on which movement pattern is ideal for the spread of a virus on a macroscopic scale, without the consideration of such influence on the microscopic scales. Simple parameters have been implemented which depend 


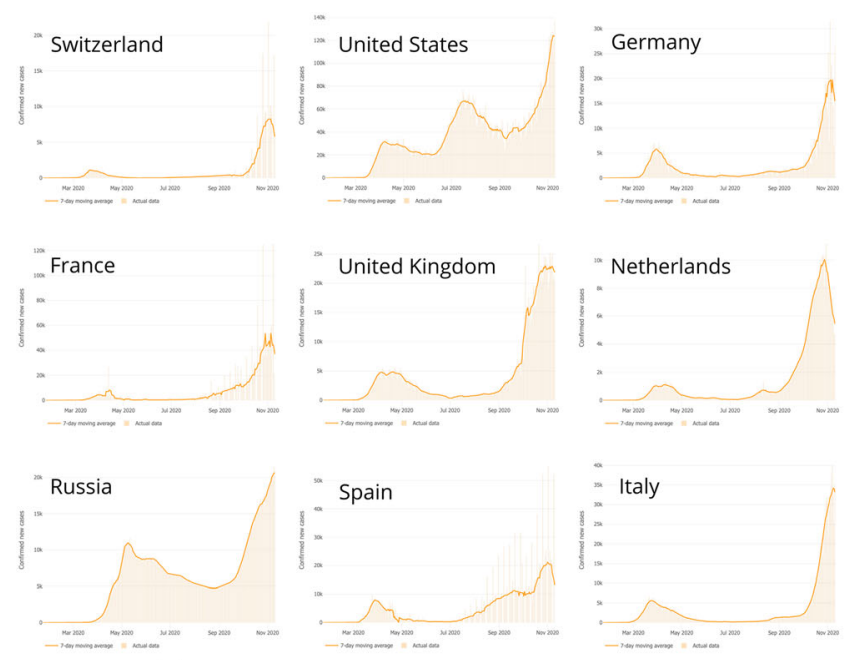

(a) Comparing data of different countries

Fig. 1 COVID-19 data

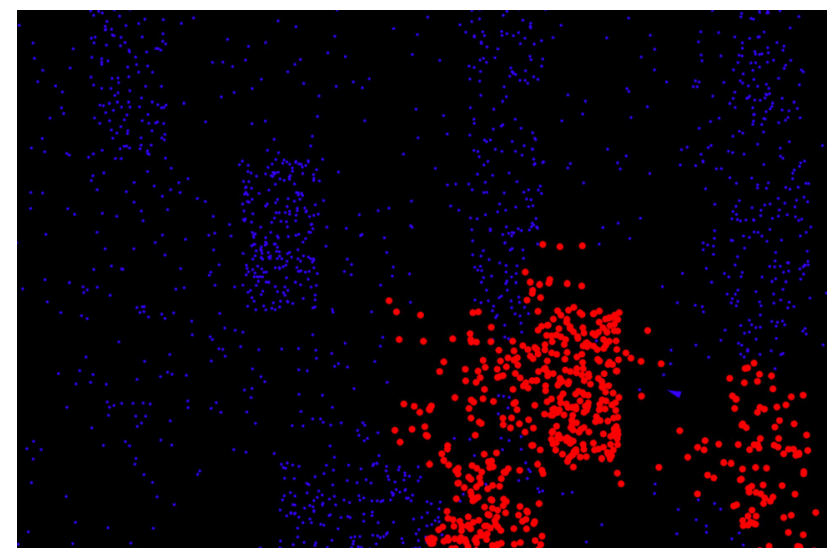

Fig. 2 Simulation

only on the stable distributions to observe simulations. These simulations are based on the logic of steering behaviour developed by Craig Reynolds [9] and Daniel Shiffman [10].

Previously, such models have been extensively studied using many parameters to govern the spread [11]. Also most human mobility models and realistic simulations which are needed for applications like SLAW [12] and SWIM [13] are based on Lévy walks. There are also other compartmental models that typically forecast the rate of spread and behavioural count of the population (such as the compartments of susceptibles, infectives and recovered in the SIR model) [14, 15].

\section{Simulation Model}

Two-dimensional canvas of dimensions $1080 \times 720$ is being considered characterised by blue dots indicative of population densities as shown in Fig. 2. This canvas may

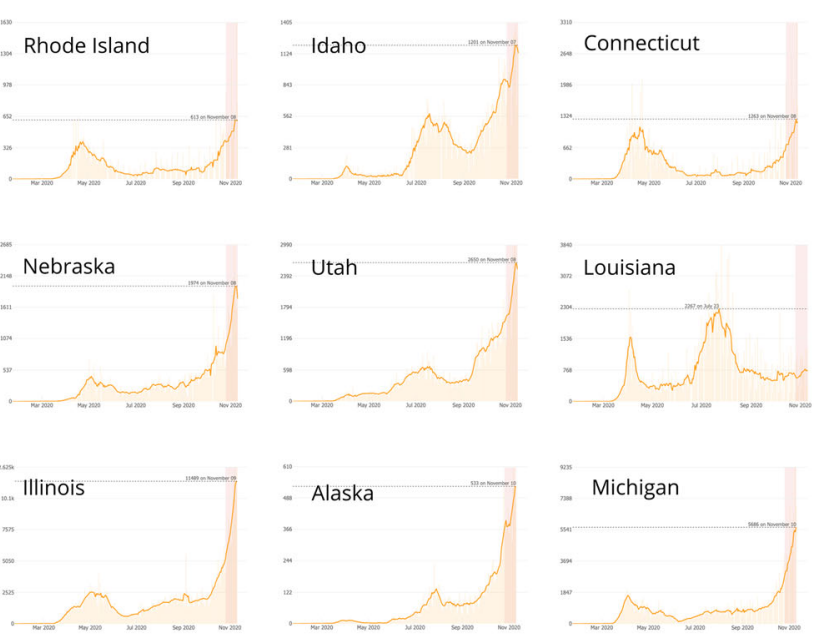

(b) Comparing data of different states

represent a continent, a country or a state where the scale invariant behaviour is observed; consecutively, the blue dots have high density value if they represent a continent and low value if they represent a state. The population is highly concentrated in some regions and sparsely spread in other. A single autonomous carrier (blue triangle) moves around randomly controlled by ND or LD spanning across the canvas infecting and spreading the virus indicated by turning the blue to red dots. There may be no direct physical significance to this carrier since the virus can spread by many different means. As the autonomous carrier moves along in a direction, it has a perceptive radius of few pixels where it infects only the blue dots within a definite boundary surrounding it. The perceptive radius imitates the realistic situation where a certain carrier can only infect a specific region around. Multiple iterations were run of a simulation with same parameters for different stable distributions to observe how the spread varies. The time taken by the autonomous carrier was obtained to infect $50 \%$ of the population and indicate it by $T_{50}$, whereas the mean time for different iterations as $\overline{T_{50}}$. They are measured in seconds (s) where the wall time for each iteration is approximately equal to $T_{50}$.

\section{Comparing Simulations of LD and ND}

Initially we studied which stable distribution is more ideal for the spread. The probability distribution function (PDF) for LD is described by 


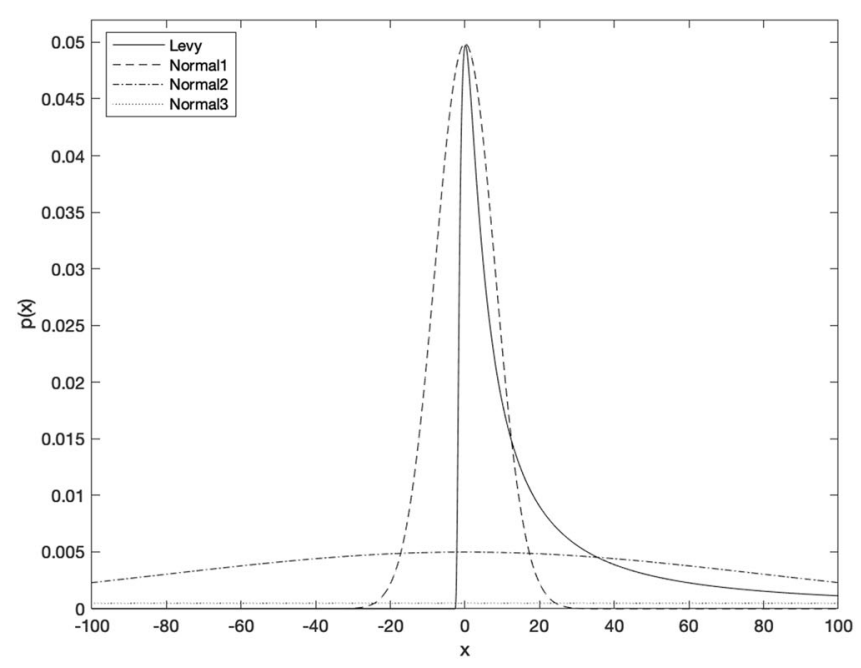

(a) Comparing Lévy and Normal1 distribution

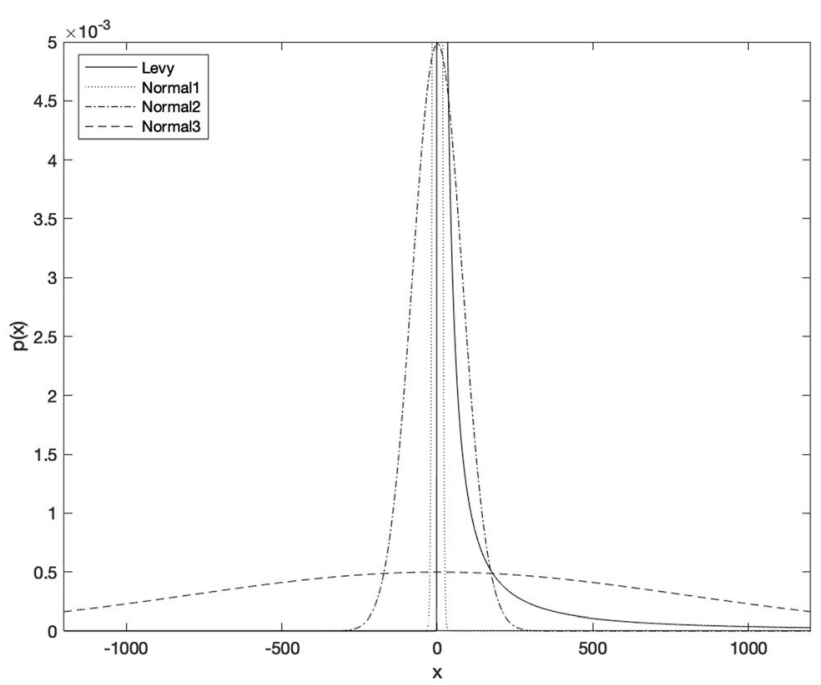

(b) Comparing Lévy and Normal3 distribution

Fig. 3 Comparing probability distributions

$$
p(x)=\sqrt{\frac{\gamma}{2 \pi(x-\delta)^{3}}} \exp \left(\frac{-\gamma}{2(x-\delta)}\right)
$$

where $\gamma$ is the scale parameter and $\delta$ is the location parameter. Similarly, ND is described by

$$
p(x)=\frac{1}{\sqrt{2 \pi \sigma^{2}}} \exp \left(\frac{-x^{2}}{2 \sigma^{2}}\right)
$$

where $\sigma$ is the standard deviation. Here $x$ represents random values in the distribution with probability $p(x)$ which are different lengths traversed by the carrier in pixels. For the LD shown in Fig. 3(a) where $\gamma=9.3$ and $\delta=-3$, we obtain $\overline{T_{50}}$ as $291 \pm 21 \mathrm{~s}$. The parameters were closed such that the peak for the PDFs are comparable. Similarly, for NDs Normal1 (N1) and Normal3 (N3) whose standard deviations are $\sigma(N 1)=8$ and $\sigma(N 3)=800$ as shown in Fig. $3 \mathrm{a}$ and $3 \mathrm{~b}$, we obtain $\overline{T_{50}}$ as $714 \pm 173 \mathrm{~s}$ and $412 \pm 57$ $\mathrm{s}$, respectively. The standard deviation was considered of $\mathrm{N} 1$ such that it is comparable with the perceptive radius of

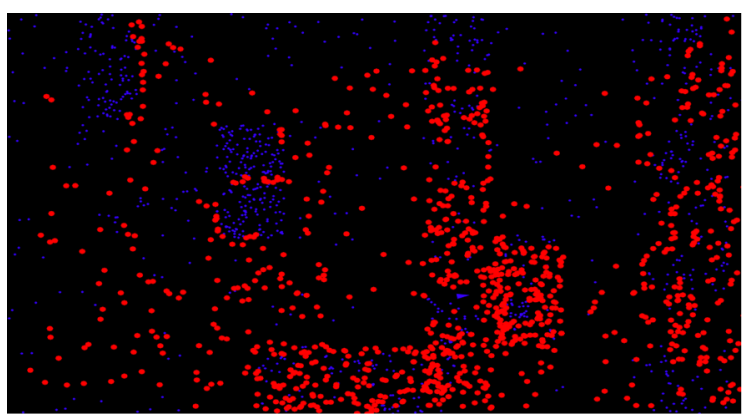

(a) Lévy walk the autonomous carrier and the standard deviation of N3 is comparable with the size of the canvas.

It was observed that the spread in LW is more patchy but spans the entire region (Fig. 4a), whereas in BW with low $\sigma$ such as N1, the spread is more thorough and confined to particular regions (Fig. $4 \mathrm{~b}$ ). It takes longer $T_{50}$ compared to $\mathrm{LW}$ and is more non deterministic. The measurement was done on $\overline{T_{50}}$ for other NDs with standard deviation ranging between $\sigma(N 1)$ and $\sigma(N 3)$. $\sigma$ of ND Normal2 (N2) is the geometric mean of $\sigma(\mathrm{N} 1)$ and $\sigma(\mathrm{N} 3)$ as shown in Fig. 3a, 3b. For ND with $\sigma$ ranging between $\sigma(\mathrm{N} 1)$ and $\sigma(\mathrm{N} 2), \overline{T_{50}}$ was obtained as $420 \pm 64$ s. $\overline{T_{50}}$ for $\mathrm{N} 2$ was obtained as $400 \pm 53$ s. Similarly, for ND with $\sigma$ ranging between $\sigma(\mathrm{N} 2)$ and $\sigma(\mathrm{N} 3), \overline{T_{50}}$ was obtained as $372 \pm 37 \mathrm{~s}$.

\section{Comparing Simulations of LD with Varying Parameters $\gamma$ and $\delta$}

The parameters for controlling LD were varied, $\gamma$ and $\delta$ to observe the change in spread by finding $\overline{T_{50}}$ in each case.

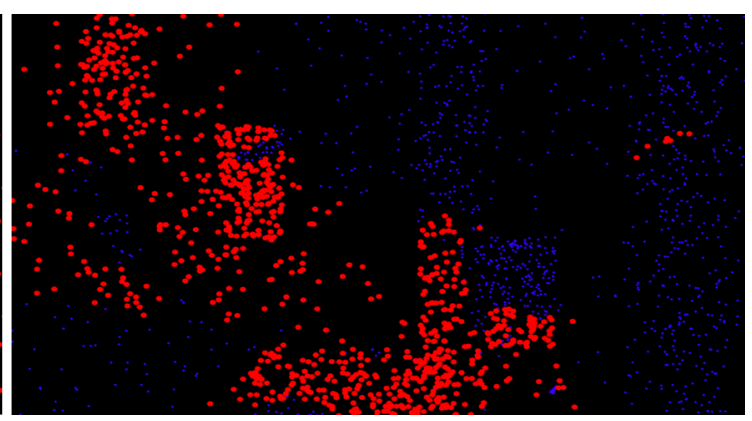

(b) Brownian walk

Fig. 4 Simulations of random walk 
Table $1 \overline{T_{50}}$ of LD with varying $\gamma$ and $\delta$

\begin{tabular}{|c|c|c|c|c|c|c|}
\hline \multirow[t]{2}{*}{$\delta$} & \multicolumn{6}{|l|}{$\gamma$} \\
\hline & $10^{-6}$ & $10^{-3}$ & $10^{-2}$ & $10^{-1}$ & $10^{0}$ & $10^{1}$ \\
\hline $10^{0}$ & $60 \pm 10 \mathrm{~s}$ & $60 \pm 7 \mathrm{~s}$ & $57 \pm 8 \mathrm{~s}$ & $58 \pm 7 \mathrm{~s}$ & $58 \pm 9 \mathrm{~s}$ & $54 \pm 7 \mathrm{~s}$ \\
\hline $10^{1}$ & $65 \pm 13 s$ & $57 \pm 9 \mathrm{~s}$ & $60 \pm 7 \mathrm{~s}$ & $60 \pm 9 \mathrm{~s}$ & $56 \pm 7 \mathrm{~s}$ & $55 \pm 7 \mathrm{~s}$ \\
\hline $10^{2}$ & $50 \pm 7 \mathrm{~s}$ & $51 \pm 5 \mathrm{~s}$ & $49 \pm 6 s$ & $49 \pm 6 s$ & $51 \pm 6 \mathrm{~s}$ & $51 \pm 7 \mathrm{~s}$ \\
\hline $10^{3}$ & $56 \pm 7 \mathrm{~s}$ & $55 \pm 7 \mathrm{~s}$ & $55 \pm 6 \mathrm{~s}$ & $54 \pm 5 \mathrm{~s}$ & $51 \pm 7 \mathrm{~s}$ & $53 \pm 7 s$ \\
\hline
\end{tabular}

Location parameter $\delta$ determines where the peak of the probability distribution function lies and scale parameter $\gamma$ determines the proportion of short steps and long steps. To study this wide range of parameter space, faster simulation rates were used since we run around 50 hours of simulation. This may affect the accuracy of measuring $\overline{T_{50}}$ but a qualitative study can be made. We display $\overline{T_{50}}$ for varying parameters by factor of 10 in Table 1 . The region of parameter space has been displayed where significant change was observed.

A deducible change could not be observed in $T_{50}$ by varying $\delta$ for each individual value of $\gamma$. However for $\delta=$ $10^{2}$ the model dimensions are ideal where steps of lengths $10^{2}$ pixels are more prominent for uniform spread, without hitting the boundary and find $\overline{T_{50}}$ to be minimum. This is also observed when studying ND simulations with $\sigma$ ranging between $80(\mathrm{~N} 2)$ and $800(\mathrm{~N} 3)$. Hitting the boundary or corners delays the spread since the carrier traverses through already infected regions and has to come back. Varying the $\gamma$ for different $\delta$, we observe that smaller $\gamma$ and $\delta$ values delay the spread where too many short steps take place compared to long steps. Long steps are necessary to cover the wide region, and also $T_{50}$ becomes more nondeterministic in this parameter space. If the carrier is stuck

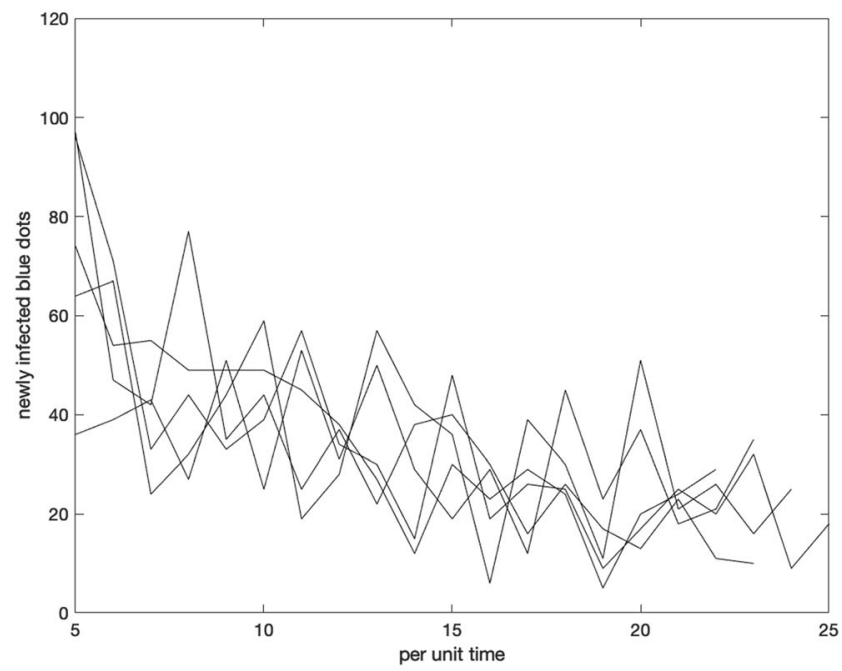

(a) LD spread for $\delta=10^{2}$ and $\gamma=10^{-6}$

Fig. 5 Newly infected blue dots per unit time for different iterations taking many short steps in a region of highly crowded blue dots $T_{50}$ value becomes low, whereas if stuck in a sparse region $T_{50}$ is high. But also too many long steps in the region of higher $\gamma$ and $\delta$ values make the spread slower than ideal, with similar behaviour not affecting $T_{50}$. The newly infected blue dots have been displayed per unit time of different iterations for $\gamma=10^{-6}$ in Fig. 5(a) and for $\gamma=10^{1}$ in Fig. 5(b). To reduce the boundary effects, we chose $\delta=10^{2}$ and observed how the spread varies for different $\gamma$. For lower $\gamma$ value, a steady decline has been observed, whereas for the higher $\gamma$ value, the fall and increase have been observed in the newly infected blue dots per unit time.

\section{Comparing Simulations of Log-Normal Distribution}

Though a log-normal distribution has similar characteristics with LD and ND in the sense that it can be expressed by sum of arbitary number of IIDs [16], it is not a stable distribution [17]. However since the interest is in distributions whose normalized sum can reproduce the original distribution, it was considered. Recent evidence suggests that individual modes of human mobility are

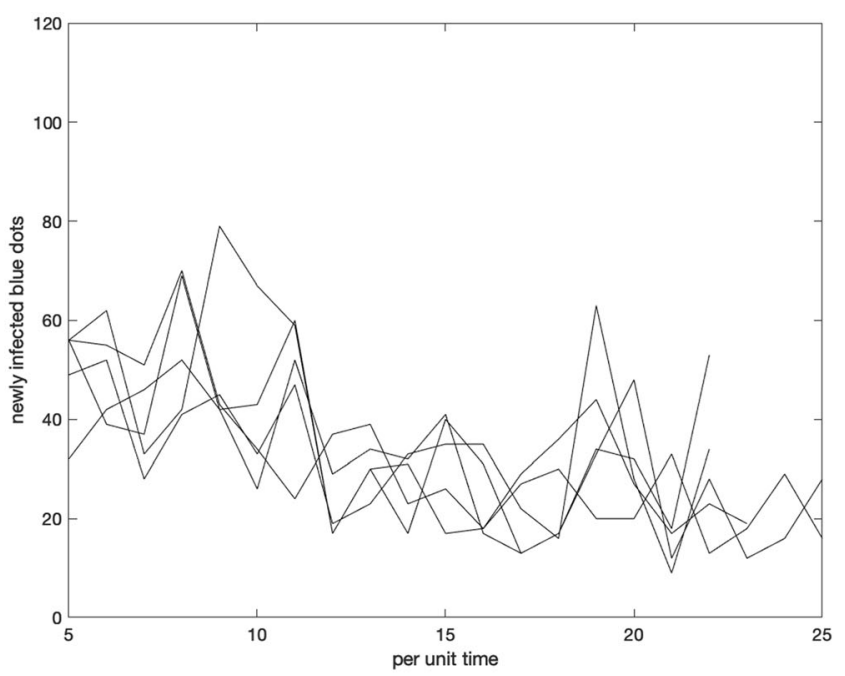

(b) LD spread for $\delta=10^{2}$ and $\gamma=10^{1}$ 
based on log-normal distribution [7]. Log-normal distribution is described by PDF

$p(x)=\frac{1}{x \sqrt{2 \pi \sigma^{\prime 2}}} \exp \left(\frac{-(\ln x)^{2}}{2 \sigma^{\prime 2}}\right)$,

and the parameter $\sigma^{\prime}$ is chosen such as the distribution is relatable to LD with heavy-tailed characteristics. For the same simulation rates used in previous section, we do not observe much difference by varying $\sigma^{\prime} \geq 10$ where it has been obtained as $\overline{T_{50}}=56 \pm 6 \mathrm{~s}, 56 \pm 8 \mathrm{~s}$ and $57 \pm 6 \mathrm{~s}$ for $\sigma^{\prime}=10,100$ and 1000 , respectively. Here, the long steps are dominating which are essential for the spread. For $\sigma^{\prime}<10$ it has been observed the PDF narrowed with limiting the number of long steps where it has been obtained as, $\overline{T_{50}}=60 \pm 13 \mathrm{~s}$ for $\sigma^{\prime}=1$.

\section{Conclusion}

Initially it was analysed for this model of simulation, Levy walk is the most ideal movement pattern for the spread of a virus. This may be related to the principle of least effort in the context of Zipf distribution [18], which have similar longrange correlations $[19,20]$. It can perhaps be seen as by following Lévy walk the autonomous carrier takes the least effort to achieve maximum spread compared to Brownian walk. Further, the study was done on how the parameters in Lévy and log-normal distributions affect the spread and how the behaviour of the spread varies. To consolidate, the random variables have been implemented from scale-invariant continuous distributions as random events causing movement of a virus to study how the spread changes. Recently, scale-invariant discrete structures like fractals were implemented to study the spread of COVID-19[21]. Implementing such selfsimilarity into other mathematical models used to study the epidemic may lead to new insights. Future studies can be tried using more realistic parameters $[11,15]$ such as restricting the movement in infected zones, implementing multiple carriers and varying the dimensions of the simulations which could imitate real world scenario.

\section{References}

1. C.T. Kello, G.D. Brown, R. Ferrer-I-Cancho, J.G. Holden, K. Linkenkaer-Hansen, T. Rhodes, G.C. Van Orden, Scaling laws in cognitive sciences. Trends Cognit Sci 14(5), 223-232 (2010)
2. Mehran Kardar, Statistical Physics of Particles (Cambridge University Press, Cambridge, 2007)

3. M. Andy, Reynolds, Current status and future directions of Lévy walk research. Biology Open 7(1) (2018)

4. D. Brockmann, L. Hufnagel, T. Geisel, The scaling laws of human travel. Nature 439(7075), 462-465 (2006)

5. M.C. González, C.A. Hidalgo, A. Barabási, Understanding individual human mobility patterns. Nature 453(7196), 779-782 (2008)

6. I. Rhee, M. Shin, S. Hong, K. Lee, S.J. Kim, S. Chong, On the Lévy-walk nature of human mobility. IEEE/ACM Trans. Network. 19(3), 630-643 (2011)

7. K. Zhao, M. Musolesi, P. Hui, W. Rao, S. Tarkoma, Explaining the power-law distribution of human mobility through transportation modality decomposition. Sci. Rep. 5(1), 9136 (2015)

8. E. Dong, H. Du, L. Gardner, An interactive web-based dashboard to track COVID-19 in real time The Lancet. Infect. Dis. 20(5), 533-534 (2020)

9. C. W. Reynolds. Steering behaviors for autonomous characters. In: Proceedings of the 1999 Game Developer's Conference, 1999

10. D. Shiffman. The nature of code. 2012

11. Abdou Moutalab Fofana, Amy Hurford, Mechanistic movement models to understand epidemic spread. Philos. Trans. R. Soc. B Biol. Sci. 372(1719), 20160086 (2017)

12. K. Lee, S. Hong, S.J. Kim, I. Rhee, S. Chong, SLAW: self-similar least-action human walk. IEEE/ACM Trans. Network. 20(2), 515-529 (2012). https://doi.org/10.1109/TNET.2011.2172984

13. Alessandro Mei and Julinda Stefa. SWIM: A simple model to generate small mobile worlds, 2009

14. W.O. Kermack, A.G. McKendrick, A contribution to the mathematical theory of epidemics. Proc. R. Soc. Lond. A 115, 700-721 (1927). https://doi.org/10.1098/rspa.1927.0118

15. W. Herbert, Hethcote, The mathematics of infectious diseases. SIAM Rev. 42(4), 599-653 (2000)

16. Olof Thorin, On the infinite divisibility of the lognormal distribution. Scandinavian Actuarial J 1977(3), 121-148 (1977)

17. X. Gao, H. Xu, D. Ye, Asymptotic behavior of tail density for sum of correlated lognormal variables. Int. J. Math. Math. Sci. 2009, 630857 (2009). https://doi.org/10.1155/2009/630857

18. George Kingsley Zipf, Human Behavior and the Principle of Least Effort: An Introduction to Human Ecology (AddisonWesley Press Inc., New York, 1949)

19. Michael F. Shlesinger, George M. Zaslavsky, Joseph Klafter, Strange kinetics. Nature 363(6424), 31-37 (1993)

20. A. Czirók, R.N. Mantegna, H.E. Shlomo, Stanley Correlations in binary sequences and a generalized Zipf analysis. Phys. Rev. E 52, 446-452 (1995)

21. C.M. Păcurar, B.R. Necula, An analysis of COVID-19 spread based on fractal interpolation and fractal dimension. Chaos Solitons Fract. 139, 110073 (2020)

Publisher's Note Springer Nature remains neutral with regard to jurisdictional claims in published maps and institutional affiliations. 